\title{
Hydrodynamique et qualité des eaux souterraines dans le bassin sédimentaire de Douala (Cameroun): cas des aquifères sur formations Quaternaires et Tertiaires
}

\author{
Adoua KOPA NJUEYA ${ }^{1,2}$, Jonathan Daniel Hervé LIKENG ${ }^{1}$ et Alexandre NONO ${ }^{1 *}$ \\ ${ }^{1}$ Département des Sciences de la Terre, Faculté des Sciences B.P. 67, Université de Dschang, \\ Dschang, Cameroun. \\ ${ }^{2}$ Département du Génie Rural, Faculté d'Agronomie et des Sciences Agricoles, B.P. 222, Dschang, Cameroun. \\ *Auteur correspondant, E-mail: alex_nono2000@yahoo.fr,njuedou@yahoo.fr
}

\section{RESUME}

La ville de Douala souffre de l'insuffisance de l'alimentation en eau potable et les populations se retournent vers les eaux des puits et forages sans garantie de qualité. Cette étude contribue à établir une relation entre la lithologie, la qualité et la dynamique des eaux souterraines. Les prospections cartographique, hydrologique, hydrogéologique et géoélectrique, les implantations et suivi de l'exécution des forages ainsi que des analyses physico-chimiques et bactériologiques des eaux ont été réalisés. La lithologie du bassin entre 0 et $100 \mathrm{~m}$ de profondeur comprend les alluvions, des sables, argiles et grès en alternance. Les données géophysiques et de foration montrent deux principaux aquifères: les nappes superficielles (de 0 à $17 \mathrm{~m}$ ) et les nappes profondes (de 20 à $73 \mathrm{~m}$ ). La minéralisation influencée par l'interaction eau-roche et la mer, évolue en moyenne entre $336,99 \mathrm{mg} / \mathrm{l}$ et 129,95 mg/l dans les nappes superficielles ; 180,08 mg/l et 71,64 mg/l dans les nappes profondes respectivement à Douala $4^{\text {ème }}$ et Douala $3^{\text {ème }}$. L'hydrochimie montre une évolution des eaux du faciès bicarbonaté calcique au faciès chloruré et sulfaté calcique et magnésien sans liaison avec l'âge des formations. Les analyses bactériologiques des eaux montrent que les nappes profondes sont de bonne qualité, tandis que les nappes superficielles abritent des coliformes totaux, coliformes et streptocoques fécaux. Les eaux des nappes superficielles évoluent d'une qualité assez bonne à médiocre à Douala $4^{\text {ème }}$ (33 à 2651 UFC/100 ml; 10 à $1700 \mathrm{UFC} / 100 \mathrm{ml}$ et 26 à $2868 \mathrm{UFC} / 100 \mathrm{ml}$ respectivement pour les coliformes totaux, coliformes et streptocoques fécaux) ou suspecte à Douala $3^{\text {ème }}$ (46 à $462 \mathrm{UFC} / 100 \mathrm{ml} ; 12$ à $350 \mathrm{UFC} / 100 \mathrm{ml}$ et 71 à 362 $\mathrm{UFC} / 100 \mathrm{ml}$ respectivement pour les coliformes totaux, coliformes et streptocoques fécaux).

(c) 2012 International Formulae Group. All rights reserved.

Mots clés: Bassin de Douala, eaux souterraines, qualité, minéralisation, hydrochimie, faciès.

\section{INTRODUCTION}

Le bassin sédimentaire de Douala fait partie de l'un des trois bassins sédimentaires côtiers que compte le Cameroun. Il se situe en bordure du golfe de Guinée entre les parallèles $3^{\circ}$ et $5^{\circ}$ de latitude Nord et possède une forme triangulaire avec une superficie d'environ
$7000 \mathrm{~km}^{2}$ (Ngueutchoua, 1996). Ce bassin est marqué par un relief relativement plat avec des altitudes très peu étalées variant entre zéro (au niveau du fleuve Wouri) et $125 \mathrm{~m}$ (au niveau de PK 35 par Bongo à $5 \mathrm{Km}$ de la route en allant vers Edéa). Il est limité: 
- au Nord et à l'Est par les affleurements du socle de la Chaîne Panafricaine d'Afrique Centrale (CPAC);

- au Nord-Ouest par les épanchements basaltiques de la Ligne du Cameroun (LC) à travers le Mont Cameroun;

- à l'Ouest et au Sud-Ouest par la côte atlantique ;

- au Sud par la côte atlantique et les formations sédimentaires du bassin de Kribi-Campo.

Les eaux souterraines et superficielles résultent principalement des eaux météoriques. Ces dernières voient leur composition être modifiée ceci en fonction de l'environnement où elles se trouvent soit sous forme de stockage ou sous forme d'écoulement (Fehdi et al., 2009). Il est aujourd'hui admis par certains auteurs (Djeuda, 1987 ; Fehdi et al., 2009; Nono et al., 2009; Mouafo, 2010), que la nature des formations géologiques ainsi que les structures géologiques influencent la dynamique et la qualité des eaux souterraines. L'un des mécanismes concourant à la modification de la composition des eaux souterraines est le phénomène d'oxydation et de précipitation de certains éléments chimiques qui se produit souvent lors de la baisse du niveau piézométrique; tel est par exemple le cas du fer comme le signale Temgoua et al. (2005). Cette modification de la composition chimique des eaux souterraines est gouvernée par cinq (05) facteurs principaux (Manga, 2001): (1) l'équilibre eau-minéral; (2) la vitesse des réactions cinétiques; (3) la composition initiale de l'eau; (4) la composition initiale de la roche servant de réservoir et (5) la vitesse d'écoulement des eaux souterraines. Cependant, signalons que d'après El Achheb et al. (2001) et Bouziane et Labadi (2009) qu'en contexte de bassin sédimentaire comme à Douala, en plus du processus d'interaction eau-roche responsable de la modification de la composition chimique des eaux souterraines, l'on note l'influence de trois (03) autres facteurs secondaires: (1) l'action de la mer (par les embruns marins, les aérosols et les biseaux salés); (2) l'existence éventuelle d'une eau profonde salée ascendante dans les zones de failles; (3) l'infiltration des eaux industrielles.

La distribution de l'eau potable au Cameroun, est assurée par la Camerounaise des eaux (CDE) qui a de la peine à satisfaire $50 \%$ des populations situées dans les grandes villes et métropoles du pays comme Douala (INS, 2005) où on note de plus en plus une recrudescence des maladies hydriques (thyphoïde, dysentérie amibienne et choléra). Cette situation (faible taux de couverture des besoins en eau potable et recrudescence des maladies hydriques) a poussé les populations ayant des revenus modestes ainsi que les autorités en charge de la ville à se retourner vers les eaux souterraines et de promouvoir la réalisation de nombreux forages équipés des pompes à motricité humaine ou des mini réseaux d'adduction afin de réduire la tranche de la population n'ayant pas accès à l'eau potable. Malheureusement, malgré ces efforts, on note encore une bonne tranche des populations qui s'approvisionnent à travers les eaux des sources ou des puits dont la qualité reste inconnue.

Dans la localité de Douala, les eaux de pluie, des puits et des forages sont sollicitées pour divers usages : boisson, lessive, ménage, breuvage des animaux, industries, etc. La majorité des ouvrages hydrauliques (puits et forages) au sein de cette agglomération est souvent effectuée sans qu'une étude de faisabilité ait été menée au départ. De même, lors de la mise en service de bon nombre de ces ouvrages, l'on fait souvent abstraction à la détermination de la qualité physico-chimique et bactériologique de ces eaux destinées à la consommation humaine; pourtant, la mauvaise qualité d'une eau de boisson est responsable des risques sanitaires chez le consommateur. Selon Kreisel (1991), les risques à long terme sont liés à la mauvaise qualité physico-chimique tandis que ceux à court terme sont liés à la qualité bactériologique; d'où il est nécessaire de 
connaître la qualité des eaux destinées à la boisson avant de les mettre à la disposition des consommateurs. L'eau est en effet un élément essentiel dans la transmission des maladies. Ceci est dû au fait qu'elle constitue un réservoir de germes (Leclerc, 1986).

Dans la ville de Douala, la majorité des puits d'approvisionnement en eau potable des populations semblent présenter une pollution bactériologique d'origine anthropique (Djuikom et al., 2009). Cette situation pousse de plus en plus les populations ainsi que les autorités à se retourner vers les eaux des forages qui dans certains cas, sont également sans garantie de qualité (Koul'a, 2006) car présentant dans plusieurs cas une pollution par le fer ou par les hydrocarbures et les gaz naturels. La construction anarchique des forages et puits sans évaluation des risques de pollution des différents niveaux aquifères, l'absence des connaissances sur la lithologie de la région et sur les techniques adaptées pour la réalisation des ouvrages hydrauliques, le manque d'un plan d'assainissement directeur connu des populations ou les décharges inopinées d'ordures contribuent à rendre inefficace les actions posées par les autorités en vue de réduire la tranche de la population n'ayant pas accès à l'eau potable. $\mathrm{Vu}$ le fait que les qualités physico-chimique et bactériologique des différents niveaux aquifères exploités dans le bassin en relation avec la lithologie sont ignorées, il se pose un problème du choix de la zone à aménager pour une adduction en eau potable, du mode de gestion de la ressource à mettre en place, et des niveaux aquifères à exploiter. Fort de ce qui précède, le présent travail constitue une innovation dans la zone pour la compréhension du système aquifère de la région. Cette étude a pour but d'améliorer la desserte en eau potable des populations situées au sein de la ville de Douala et de faciliter une gestion durable de la ressource en eau en termes de choix de la zone et de la ressource à aménager ainsi que du mode de gestion à implémenter. Plus spécifiquement, il est question pour nous d'essayer d'établir une relation entre la lithologie, les structures géologiques et le phénomène de potabilisation des ressources en eaux souterraines dans la ville de Douala en général et dans les arrondissements de Douala $3^{\text {ème }}$ et de Douala $4^{\text {ème }}$ en particulier.

\section{MATERIEL ET METHODES \\ Les sites d'étude}

Les sites d'étude (Figure 1) sont localisés au sein du bassin sédimentaire de Douala et sont représentés par des unités au sein des arrondissements de Douala $3^{\text {ème }}$ et de Douala $4^{\text {ème }}$. Le secteur de Douala $3^{\text {ème }}$ est localisé entre les coordonnées $4^{\circ} 00^{\prime}-$ $04^{\circ} 02^{\prime} 30^{\prime \prime} \mathrm{N}$ et $09^{\circ} 45^{\prime} 00^{\prime}{ }^{\prime}-09^{\circ} 47^{\prime} 30^{\prime \prime} \mathrm{E}$ tandis que celui de Douala $4^{\text {ème }}$ est compris entre les coordonnées $04^{\circ} 03^{\prime}-04^{\circ} 07^{\prime} \mathrm{N}$ et $09^{\circ} 37^{\prime}-09^{\circ} 41^{\prime} E$. Les deux secteurs d'étude sont caractérisés par un relief quasi-plat avec des altitudes comprises entre 0 et $20 \mathrm{~m}$ à Douala $4^{\text {ème }}$ et entre 0 et $60 \mathrm{~m}$ à Douala $3^{\text {ème }}$ où il est peu vallonné avec des vallées en $U$ ou en V. Ces secteurs d'étude font partie intégrante de deux grands bassins versants que sont respectivement le Wouri et la Dibamba.

\section{Milieu physique Le climat}

Il est de type équatorial humide côtier, influencé par la mer. Cependant, la proximité $\mathrm{du}$ mont Cameroun (4100 m d'altitude) influence également ce climat qui devient très pluvieux. Il s'agit d'un climat particulier, marqué par la quasi permanence des pluies. On distingue deux principales saisons: une saison de pluie longue d'environ 9 mois (de Mars à Novembre) et une saison relativement sèche courte d'environ 3 mois (de Décembre à Février). La température moyenne annuelle oscille autour de $27,5^{\circ} \mathrm{C}$. Le mois d'Août est le mois le plus froid avec une température moyenne mensuelle oscillant autour de 25,5 ${ }^{\circ} \mathrm{C}$ tandis que le mois de Février est le mois le plus chaud avec une température moyenne mensuelle oscillant autour de $28,9{ }^{\circ} \mathrm{C}$. 


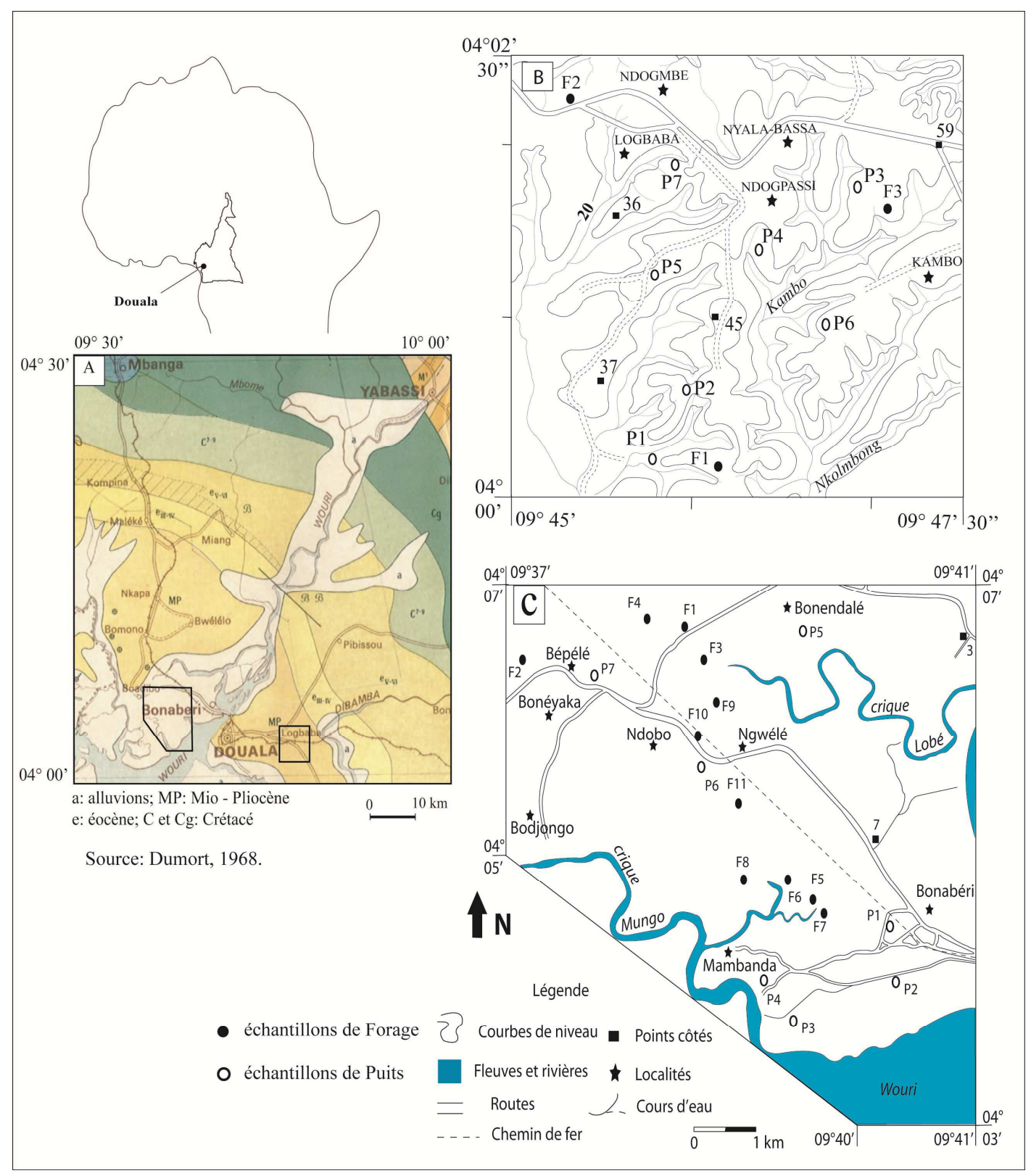

Figure 1: Localisation du Cameroun dans l'Afrique suivi de la carte géologique du bassin de Douala ressortant la position des deux secteurs d'étude (A) et des secteurs d'étude faisant ressortir les différents points d'échantillonnage à Douala $3^{\text {ème }}(\mathrm{B})$ et Douala $4^{\text {ème }}(\mathrm{C})$.

\section{L'hydrographie}

Le réseau hydrographique au sein des zones d'étude est très dense et montre des cours d'eaux d'ordre 4 qui sont méandriformes ou rectilignes, définissant un réseau dendritique à subparallèle. Cette présentation du réseau hydrographique permet de définir un potentiel hydrologique et hydrogéologique important dans la région.

\section{La géologie}

Sur le plan géologique, les travaux de nombreux auteurs (Njike, 1984; Regnoult, 1986; Ngueutchoua, 1996) mettent en exergue un certain nombre de formations qui 
affleurent ou qui ont été traversées par des forages pétroliers et/ou reconnues à partir de la sismique. Ainsi, la lithologie du bassin de Douala présente du haut vers le bas plusieurs formations regroupées suivant leurs âges de dépôt: sédiments Quaternaires, Tertiaires et Secondaires.

(1) Les sédiments du Quaternaire sont représentés dans le bassin de Douala par les formations du Wouri. C'est une unité non consolidée, sableuse avec intercalations de silts et d'argiles devenant silteuses en surface. Cette formation repose en discordance sur toutes les séries antérieures qu'elle ravine par endroit. Les dépôts ici sont de type fluviodeltaïque. Les sables sont grossiers, à litages généralement entrecroisés et fréquemment intercalés de niveaux indurés ferrallitisés (Martin, 1979). Les études de Ngueutchoua (1996) montrent sur le plan minéralogique l'existence des calcaires avec des carbonates de calcium ayant des teneurs augmentant avec la profondeur ainsi que les argiles représentées par la kaolinite, la smectite et l'illite (suivant leur ordre d'abondance de la surface vers la profondeur).

(2) Les sédiments du Tertiaire sont représentés par les formations de Nkapa, de Souellaba et de Matanda. La formation de Matanda date du Miocène et est caractérisée surtout par des dépôts deltaïques avec des lentilles de sable. La stratification est faite d'une prédominance des sables grossiers à la base et de sables fins au sommet avec intercalations d'argiles bariolées continentales. La formation de Souellaba date de l'Oligocène. Elle est sableuse et en discordance avec la formation de Nkapa. Elle comprend des argiles noires ou brunes avec quelques petits bancs sableux, des sables argileux ainsi que des sables fins et des graviers alternant avec des argiles plastiques. La formation de Nkapa datée de l'Eocène et du Paléocène, comprend deux membres hétérogènes (Bolondo et Dizangué) qui correspondent à une remontée eustatique oscillante sur le socle quasi-pénéplainé. Cette formation est constituée de grès arkosiques montrant occasionnellement des couches de quartz avec des intercalations argilo-calcaires qui deviennent prédominantes vers la surface. Bolondo appartient au domaine franchement marin et Dizangué représente le domaine continental; les deux sont séparés par les dépôts clastiques littoraux au niveau de la marge du bassin de Douala (Regnoult, 1986).

(3) Les formations secondaires sont représentées par la formation de Logbaba et celle du Mundeck. La formation de Logbaba date du Cénomanien et elle repose en discordance ou en concordance sur les grès de base selon que la surface d'érosion existe ou non (Dumort, 1968; Njike, 1984). Elle est essentiellement constituée de grès, de sables et d'argiles sableuses fossilifères. La formation des grès de base ou du Mundeck date de l'Albo-aptien. Elle est en discordance sur le socle cristallin. Elle est bien exposée dans la partie Nord du bassin de Douala, en particulier près de Mundeck dans la vallée du Mungo où elle affleure sous une faible épaisseur du sol (Njike, 1984).

Les sols

Ils présentent une diversité marquée par la dominance des sols ferrallitiques, des sols hydromorphes, des sols peu évolués et les sols minéraux bruts (Hieng, 2003). Parmi les sols ferrallitiques, on note la dominance des sols ferrallitiques jaunes dérivés des roches sédimentaires sableuses et sablo-argileuses. La teneur en bases de ces sols est très faible et leur $\mathrm{pH}$ est acide (en moyenne 5,5).

\section{Les populations et l'activité économique}

Fort de son potentiel humain de 1931 977 habitants (avec 646347 habitants à Douala $3^{\text {ème }}$ et 250626 habitants à Douala $4^{\text {ème }}$ ), soit environ $77 \%$ de la population générale de la région du Littoral, la ville de Douala constitue la ville la plus peuplée de la région du littoral (BUCREP, 2010). Cette population est cosmopolite. L'activité économique est très intense dans cette ville et se trouve être principalement le commerce, le 
transport (aérien, terrestre et maritime) et l'industrie. Outre ces trois activités, l'agriculture et la pêche ne sont pas en reste. La diversité des activités économiques, la démographie galopante ainsi que la dynamique des populations sont susceptibles d'influencer la qualité des eaux souterraines tant sur le plan physico-chimique que bactériologique. Signalons que dans les secteurs d'étude, les puits ne sont pas souvent aménagés et jouxtent les latrines ou les points d'évacuation des eaux usées des usines ou des ménages. Ceci est dû à une occupation non contrôlée des terres.

\section{Méthodes d'étude}

La présente étude a été menée tant sur le terrain qu'en laboratoire. Sur le terrain, une prospection hydrologique et géophysique a permis respectivement de déterminer le potentiel hydrologique de la région et les points favorables aux implantations des forages. Une analyse morphostructurale et linéamentaire a été menée afin d'établir une relation entre le modelé du relief et l'action de la tectonique. La description des coupes lithologiques des puits ou des broyats «cuttings» récoltés au cours de la réalisation des forages a abouti à la détermination de la nature des formations géologiques et la nature du matériau aquifère par la méthode manuelle sur le terrain. Les caractéristiques hydrodynamiques ont été obtenues après interprétation des courbes de remonté tracées à partir des données des essais de pompage. En ce qui concerne la localité de Douala $3^{\text {ème }}$, un seul essai a été réalisé (forage $F_{2}$ ) tandis qu'à Douala $4^{\text {ème }}$, on a réalisé 8 essais de pompage (forages $\mathrm{F}_{1}, \mathrm{~F}_{2}, \mathrm{~F}_{3}, \mathrm{~F}_{4}, \mathrm{~F}_{7}, \mathrm{~F}_{8}, \mathrm{~F}_{10}$ et $\left.F_{11}\right)$. Les caractéristiques déterminées concernent la transmissivité (T) et le coefficient de perméabilité (K). Le prélèvement des échantillons d'eaux pour analyses et les mesures des paramètres physiques in - situ ont été effectués.

En ce qui concerne l'aspect géophysique, nous avons utilisé la méthode électrique suivant la norme Schlumberger. Le principe consiste à envoyer dans le sol, grâce à des électrodes $\mathrm{A}$ et $\mathrm{B}$, un courant d'intensité I, puis, on mesure la différence de potentiel $\Delta \mathrm{V}$, produite par effet ohmique, entre deux électrodes de réception $\mathrm{M}$ et $\mathrm{N}$. Ce moyen, permet de déterminer les hétérogénéités (i.e les anomalies conductrices ou résistantes) induisant des variations de résistivité, dues à des causes naturelles (changement de nature ou de faciès de la roche, failles, fractures, zones broyées, poches de dissolution, avens, grottes) ou artificielles (galeries, conduites). Les valeurs des résistivités apparentes sont obtenues par des mesures directes à l'aide d'un résistivimètre (marque SYSCALR2 IRIS - INSTRUMENT).

$\mathrm{Au}$ laboratoire, le travail a consisté à effectuer une modélisation des courbes de sondages géoélectriques à l'aide du logiciel Géo-électromod/Excell pour faciliter les interprétations. Les études cartographiques des deux secteurs d'étude se sont faites à l'aide des photographies aériennes et des cartes topographiques au 1/50000. Ces études ont consisté en la confection des cartes linéanmentaires et des coupes lithologiques des différents ouvrages (puits et forages).

Les analyses physico-chimiques des eaux se sont effectuées dans deux laboratoires: le Laboratoire de Botanique Appliquée (LABOA) de la Faculté des Sciences et le Laboratoire d'Analyse des Sols et de Chimie de l'Environnement (LABASCE) de la Faculté d'Agronomie et des Sciences Agricoles de l'Université de Dschang. Ces analyses ont porté sur deux échantillons d'eau de pluie (un dans chaque secteur), quatorze (14) échantillons d'eau de puits (soit sept échantillons dans chaque secteur d'étude) et sept (07) échantillons d'eau de forage (soit 3 échantillons à Douala $3^{\text {ème }}$ et 04 à Douala $4^{\text {ème }}$ ). Ces analyses physico-chimiques concernent la conductivité, les solides totaux dissous (STD), la turbidité et la concentration des nitrates, des sulfates, du fer, des phosphates, du calcium, du 
magnésium, du potassium, du sodium, des chlorures et des bicarbonates présents dans l'eau. La méthode spectrométrique d'émission atomique a été utilisée pour la détermination $\mathrm{du}$ sodium et du potassium, la méthode complexométrique pour le calcium et le magnésium, la méthode colorimétrique pour le fer, les nitrates, les sulfates et les phosphates, la méthode potentiométrique pour les chlorures et la méthode $\mathrm{pH}$-métrique pour les bicarbonates ainsi que les carbonates.

Les analyses microbiologiques (bactériologiques) des échantillons d'eau des puits et des forages se sont effectuées au LABOA. Ces analyses microbiologiques concernent la détermination du taux de coliformes fécaux, des coliformes totaux et des streptocoques fécaux. Les techniques utilisées pour la détermination de ces paramètres sont celles de la fermentation en tubes multiples (pour la détermination présomptive des coliformes totaux) et de la membrane filtrante (pour la détermination des streptocoques fécaux et des coliformes fécaux ainsi que totaux).

\section{RESULTATS}

\section{Prospections Hydrologique et Géologique}

Les résultats de ces prospections révèlent que la productivité des ouvrages (puits et forages), est fonction de leur position topographique (sur une crête, un flanc ou dans un bas fond), de leur rapprochement d'un collecteur (rivière) et du degré hydrologique $\mathrm{du}$ collecteur (ordre 1 ou ordre 4). Dans l'arrondissement de Douala $3^{\text {ème }}$, les puits situés sur les flancs et au sommet des collines sont de grande profondeur (jusqu'à $20 \mathrm{~m}$ ) et tarissent en pleine saison sèche tandis qu'à Douala $4^{\text {ème }}$, on ne rencontre pas ce problème quel que soit la saison; cependant la profondeur des puits ne dépasse guère les 13 m.

En ce qui concerne les études des profils pédologiques on note dans les niveaux superficiels (entre 3 et $15 \mathrm{~m}$ ), la présence d'un niveau d'accumulation $\mathrm{du}$ fer sous forme oxydée se présentant de manière générale dans les sables fins à moyens latéritisés de couleur rouge brique. $\mathrm{La}$ profondeur d'accumulation du fer dans le profil pédologique de Douala varie suivant les localités, et ce en fonction de la topographie. C'est ainsi par exemple que des sables fins latéritisés sont localisés entre 0 et $5 \mathrm{~m}$ dans le profil des forages $F_{1}$ et $F_{10}$ et entre 10 et $15 \mathrm{~m}$ de profondeur dans le profil de $F_{2}$ à Douala $4^{\text {ème }}$; tandis qu'ils se trouvent entre 10 et $15 \mathrm{~m}$ dans la coupe de $F_{2}$ et entre 25 et $30 \mathrm{~m}$ de profondeur dans la coupe $\mathrm{F}_{3}$ à Douala $3^{\text {ème }}$.

Les formations géologiques sont caractérisées de manière générale par les sables, les argiles et les grès en alternance.

Les argiles rencontrées dans les deux localités sont faites de particules fines et collantes. Ces particules forment des boudins qui ne se rompent pas entre les mains lorsqu'ils sont imbibés d'eau. De part la couleur de ces argiles, on distingue deux types à Douala $3^{\text {ème }}$ (les argiles grises et les argiles bariolées) tandis qu'elles sont de trois types à Douala $4^{\text {ème }}$ (les argiles grises, les argiles bariolées et les argiles noires). Les argiles grises contiennent quelques particules de sable, fins et sont gorgées d'eau; les argiles bariolées présentent quant à elles de multiples taches (rouge, grise, jaunâtre, blanche et beige) et se présentent dans certains cas sous forme de plaquettes. Les argiles noires sont sombres et disposées sous forme de plaquettes contenant très peu d'eau.

Les sables montrent trois classes: les sables fins $(0,09<\varnothing<0,2 \mathrm{~mm})$, moyens $(0,3$ $<\varnothing<0,5 \mathrm{~mm})$ et grossiers $(0,6<\varnothing<2 \mathrm{~mm})$. Ces formations constituent les principaux aquifères rencontrés entre 05 et $17 \mathrm{~m}$ de profondeur pour les aquifères superficiels et entre 20 et $73 \mathrm{~m}$ de profondeur pour les aquifères profonds. Au sein de ces sables, on remarque la présence du quartz et de certains minéraux ferromagnésiens tels que les paillettes de biotite. Les sables fins sont de couleur grise à rougeâtre ou rouille rappelant parfois les sables obtenus des roches granito- 
gneissiques observées dans les hautes terres de l'Ouest. Ces sables constituent l'essentiel des aquifères des nappes superficielles et présentent de nombreuses variantes tels que les sables fins argileux ou latéritisés. Au sein de ces sables fins, on rencontre souvent quelques particules de pouzzolanes (cas de la localité de Nyalla à Douala $3^{\text {ème }}$ ) ou des sables fins calcaires (entre 14 et $25 \mathrm{~m}$ de profondeur) réagissant à l'acide chlorique (cas de la localité de Bonendale par Bonabérie à Douala $\left.4^{\text {ème }}\right)$. Les sables moyens sont également de couleur grise et se distinguent des sables fins de par leur taille légèrement supérieure. $\mathrm{Au}$ sein de ces sables moyens, on rencontre des galets de quartz lorsque ces derniers constituent des aquifères (cas de $\mathrm{F}_{4}, \mathrm{~F}_{9}$ et $\mathrm{F}_{10}$ à Douala $4^{\text {ème }}$ par exemple). Enfin, les sables grossiers sont de couleur grise. Ces sables grossiers sont souvent argileux et présentent dans certains cas, des particules de la taille des graviers $(6<\varnothing<20 \mathrm{~mm})$. Ces sables grossiers constituent l'essentiel des formations aquifères des nappes profondes.

Les grès se rencontrent dans le profil de la coupe lithologique de Nyalla (à Douala $3^{\text {ème }}$ ) où ils se situent entre 21 et $27,5 \mathrm{~m}$ de profondeur environ et dans le profil de la coupe lithologique $\left(\mathrm{F}_{3}\right)$ de Bonendale (à Douala $4^{\text {ème }}$ ) où ils se situent entre 65 et $74 \mathrm{~m}$ de profondeur environ. Ils sont constitués par des particules de sables fins à moyens consolidées par un ciment argileux de couleur légèrement grise et un ciment légèrement ferrugineux entrainant le passage des grès de la couleur grise à celle rosâtre. Au sein de ces grès l'on remarque également la présence de petits galets ayant la taille approximative de $0,5 \mathrm{~cm}$.

\section{Morphostructurale et données de foration}

L'analyse morphostructurale montre un relief contrasté, en ce sens qu'à Douala $3^{\text {ème }}$ le relief est vallonné avec des vallées parfois en $\mathrm{V}$ et rectilignes de direction $\mathrm{NE}-\mathrm{SW}$ et des vallées étalées avec des cours d'eau méandriformes. Cependant à Douala $4^{\text {ème }}$, on constate que le Wouri présente un écoulement suivant un axe structural NE - SW. L'analyse des photographies aériennes montre dans le secteur de Douala $3^{\text {ème }}$, un réseau de fractures ayant une direction principale de fracturation qui est $\mathrm{N} 70^{\circ}$ à $\mathrm{N} 80^{\circ} \mathrm{E}$ (Figure 2). Il existe une direction secondaire qui n'est pas négligeable à savoir $\mathrm{N} 30^{\circ}$ à $\mathrm{N} 40{ }^{\circ} \mathrm{E}$. Ces deux directions correspondent respectivement aux directions des grands accidents de la CPAC au Cameroun que sont la faille de la Sanaga (SF) et le Cisaillement Centre Camerounais (CCC). La seconde direction obtenue (N 30 à N $\left.40{ }^{\circ} \mathrm{E}\right)$ correspondrait également à la direction majeure de la Ligne du Cameroun (LC).

Les résultats de foration en concordance avec les résultats de géophysique dans la localité de Douala $3^{\text {ème }}$ et ceux de Douala $4^{\text {ème }}$ révèlent un système aquifère constitué majoritairement de 02 principaux aquifères dont l'un superficiel à nappe libre et l'autre profond et généralement à nappe captive. Dans la localité de Douala $3^{\text {ème }}$, les aquifères superficiels vont en général de 1 à $17 \mathrm{~m}$ tandis que ceux de Douala $4^{\text {ème }}$ vont jusqu'à $13 \mathrm{~m}$ de profondeur. Quant aux aquifères profonds, ils se localisent entre 20 et $40 \mathrm{~m}$ de profondeur à Douala $3^{\text {ème }}$ et sont constitués majoritairement de sable fins à moyens et parfois de grès ferrugineux. Cependant à Douala $4^{\text {ème }}$, ces aquifères profonds se localisent entre 38 et $73 \mathrm{~m}$ de profondeur et sont constitués majoritairement des sables moyens à grossiers souvent graveleux ou argileux. Ces aquifères secondaires ont une épaisseur moyenne de 9 $\mathrm{m}$ à Douala $3^{\text {ème }}$ exception faite au forage $F_{2}$ $(19 \mathrm{~m})$, tandis qu'à Douala $4^{\text {ème }}$, ils ont une épaisseur moyenne de $21 \mathrm{~m}$.

Il ressort de l'analyse des courbes de remonté des essais de pompage, que les formations aquifères des niveaux captés présentent une transmissivité moyenne de $0,325 \mathrm{~m}^{2} / \mathrm{s}$ à Douala $3^{\text {ème }}$ et de $0,193 \mathrm{~m}^{2} / \mathrm{s}$ à Douala $4^{\text {ème }}$. De même, ces formations ont un très bon degré de perméabilité avec une 
moyenne de coefficient de perméabilité égale à $2,7 \times 10^{-2} \mathrm{~m} / \mathrm{s}$. Ces données expliqueraient le fait qu'on ait des débits d'exploitation de forage allant de 3 à plus de $5 \mathrm{~m}^{3} / \mathrm{h}$.

\section{Hydrochimie et hydrogéochimie}

Les résultats des analyses physicochimiques (Tableau 1) montrent des variations notables lorsqu' on passe des eaux de pluie aux eaux de forage en passant par les eaux des puits. $\mathrm{Au}$ regard des valeurs moyennes obtenues, il ressort un comportement similaire des eaux dans les deux localités vis-à-vis des différents paramètres. C'est ainsi que la conductivité, la TDS, la température et la turbidité montrent des valeurs croissantes lorsqu'on passe des eaux de pluies aux eaux des puits; puis ces valeurs décroissent lorsqu'on passe des eaux des puits aux eaux des forages. Seul le $\mathrm{pH}$ montre un comportement inverse.

Le positionnement des différentes catégories d'eaux dans le diagramme de Piper, fait ressortir trois faciès (Figure 3):

- le faciès bicarbonaté calcique correspondant à celui des eaux de pluie ;

- le faciès bicarbonaté calcique et magnésien qui correspond à celui des eaux de puits; et

- le faciès chloruré et sulfaté calcique et magnésien correspondant aux eaux des forages.

Le triangle des cations montre que la quasi-totalité des eaux (pluie, nappe superficielle et nappe profonde) sont riches en calcium et magnésium.

Le triangle des anions quant à lui, montre deux comportements bien distincts: les eaux de puits et de pluie se retrouvent dans le domaine des eaux bicarbonatées et sont principalement riches en bicarbonate, chlorure et nitrates, tandis que les eaux de forage des deux localités ne présentent aucun anion dominant.

Le Tableau 2 ressort les rapports existant d'une part entre les alcalins et les alcalinoterreux et d'autre part entre les alcalins ainsi que les alcalinoterreux pris chacun individuellement. Les eaux des deux zones ont des rapports $\mathrm{Mg}^{2+} / \mathrm{Ca}^{2+}$ inférieurs à l'unité. De plus, ce rapport augmente progressivement avec la profondeur; ce qui signifie que de la surface en profondeur, les ions $\mathrm{Ca}^{2+}$ ont tendance à être fixés par les argiles tandis que les ions $\mathrm{Mg}^{2+}$ ont tendance à être libérés progressivement.

Le rapport alcalinoterreux et alcalin donne des valeurs supérieures à l'unité quelque soit le type d'échantillon.

Le rapport entre les alcalins montre une baisse dans l'ensemble des deux zones lorsqu'on passe des eaux de pluie aux eaux de puits. Cependant, lorsqu'on passe des eaux de puits aux eaux de forage dans les deux localités, on constate une augmentation de ce rapport qui à Douala $3^{\text {ème }}$ est supérieure à l'unité.

L'indice d'échange des bases (I.E.B) définit comme étant le rapport entre les ions échangés et les ions de même nature primitivement existant dans l'eau, permet de définir le sens des échanges ioniques entre l'eau et les terrains encaissants. Lorsque : l'I.E.B = 0 il n'y a pas d'échange ;

1'I.E.B $<0$ les ions $\mathrm{Ca}^{2+}$ et $\mathrm{Mg}^{2+}$ sont échangés par les ions $\mathrm{Na}^{+}$et $\mathrm{K}^{+}$;

l'I.E.B > 0 les ions $\mathrm{Na}^{+}$et $\mathrm{K}^{+}$sont échangés par les ions $\mathrm{Ca}^{2+}$ et $\mathrm{Mg}^{2+}$.

Les valeurs de l'I.E.B obtenues dans le cadre de cette étude sont positives dans l'ensemble.

\section{Caractérisation bactériologique des eaux}

L'analyse de la qualité microbiologique des eaux de forage dans les deux localités montre que ces dernières sont exemptes de coliformes fécaux et totaux ainsi que des streptocoques fécaux. Cependant, il n'en est pas de même pour les eaux des puits qui montrent des résultats très contrastés (Tableau 3), lorsqu'on passe de la saison de pluie (Août) à la saison sèche (Février). 

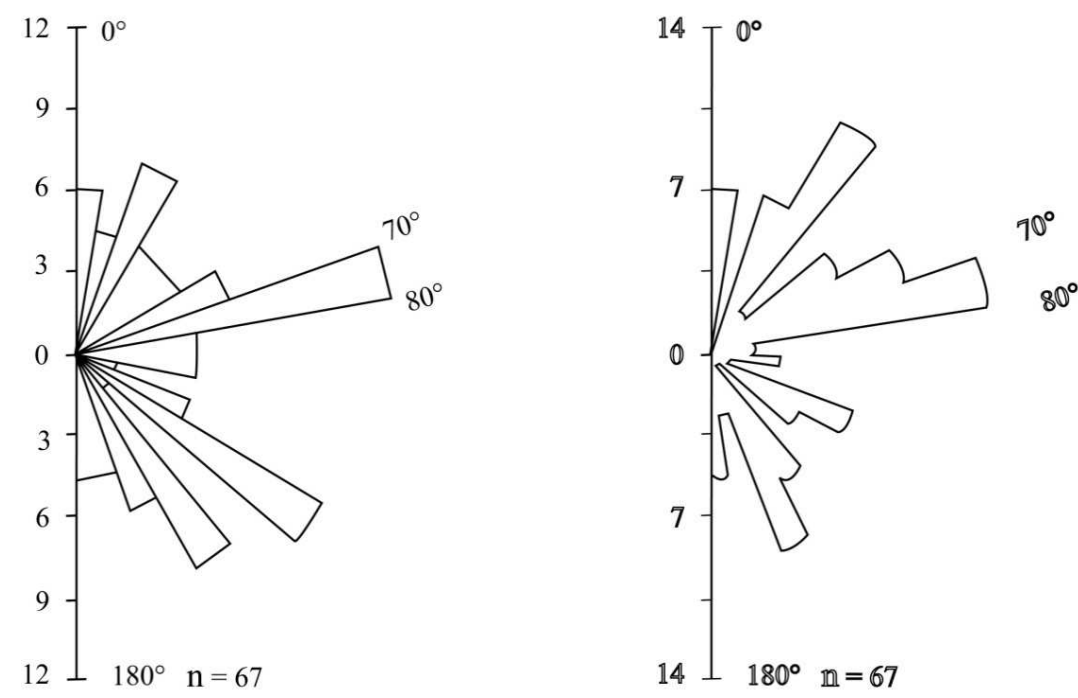

Figure 2: Rosaces de fréquences des directions (à gauche) et des longueurs cumulées (à droite) des fractures à Douala $3^{\text {ème }}$.

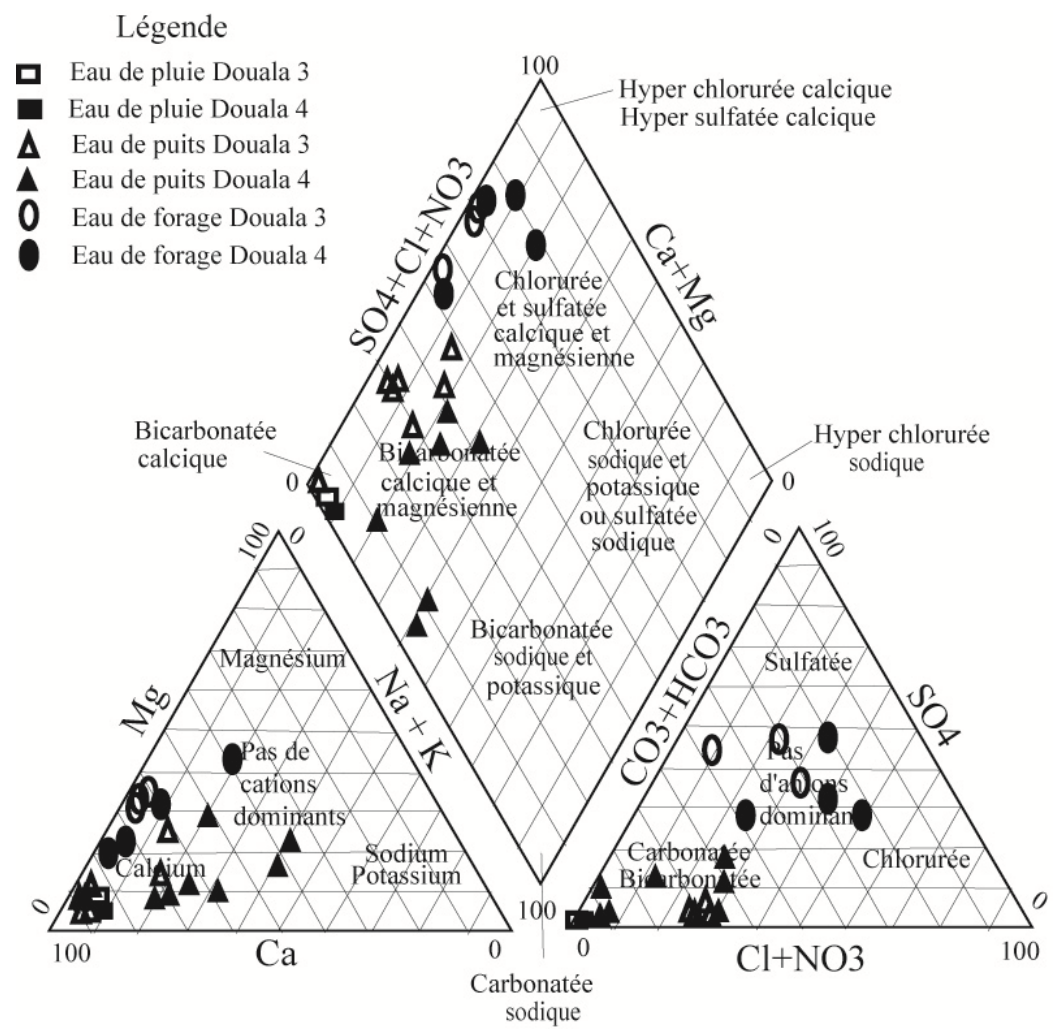

Figure 3: Diagramme de Piper donnant les faciès des différentes catégories d'eaux prélevées en fonction des localités. 


\section{A. KOPA NJUEYA et al. / Int. J. Biol. Chem. Sci. 6(4): 1874-1894, 2012}

Tableau 1: Résultats des analyses physico-chimiques des différentes catégories d'eaux analysées dans chaque localité.

\begin{tabular}{|c|c|c|c|c|c|c|c|c|c|c|c|c|c|c|c|c|c|}
\hline \multicolumn{2}{|c|}{ Echantillon } & $\begin{array}{c}\text { Conductivité } \\
(\mu \mathrm{S} / \mathrm{cm})\end{array}$ & $\begin{array}{c}\text { TDS } \\
(\mathrm{mg} / \mathrm{l})\end{array}$ & $\begin{array}{c}\text { Température } \\
\left({ }^{\circ} \mathrm{C}\right)\end{array}$ & pH & $\begin{array}{c}\text { Turbidité } \\
\text { (F.T.U) }\end{array}$ & $\begin{array}{l}\mathrm{Ca}^{2+} \\
\mathrm{mg} / \mathrm{l}\end{array}$ & $\begin{array}{l}\mathrm{Mg}^{2+} \\
\mathrm{mg} / \mathrm{l}\end{array}$ & $\begin{array}{l}\mathrm{Na}^{+} \\
\mathrm{mg} / \mathrm{l}\end{array}$ & $\begin{array}{c}\mathrm{K}^{+} \\
\mathrm{mg} / \mathrm{l}\end{array}$ & $\begin{array}{c}\mathrm{Cl}^{-} \\
\mathrm{mg} / \mathrm{l}\end{array}$ & $\begin{array}{l}\mathrm{SO}_{4}{ }^{2-} \\
\mathrm{mg} / \mathrm{l}\end{array}$ & $\begin{array}{l}\mathrm{NO}_{3}^{-} \\
\mathrm{mg} / \mathrm{l}\end{array}$ & $\begin{array}{c}\mathrm{HCO}_{3}^{-} \\
\mathrm{mg} / \mathrm{l}\end{array}$ & $\begin{array}{l}\mathrm{PO}_{4}{ }^{3-} \\
\mathrm{mg} / \mathrm{l}\end{array}$ & $\begin{array}{c}\mathrm{Fe} \\
\mathrm{mg} / \mathrm{l}\end{array}$ & $\begin{array}{l}\text { Min } \\
\text { mg/l }\end{array}$ \\
\hline \multirow{13}{*}{ 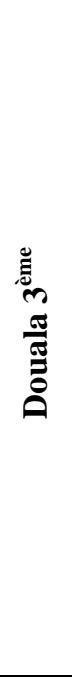 } & Pl 1 & 26,2 & 13,3 & 26,6 & 7,8 & $\mathbf{0 , 0}$ & 12,7 & 0,7 & 0,6 & 0,9 & nd & $\mathbf{0 , 0}$ & 0,7 & 36,6 & $\mathbf{0 , 0}$ & $\mathbf{0 , 0 0}$ & 27,2 \\
\hline & P1 & 320,0 & 142,0 & 26,7 & 6,2 & 0 & 24,4 & 1,7 & 3,2 & 7,4 & 33,7 & 17,0 & 5,7 & 122,0 & 0,0 & 0,02 & 246,3 \\
\hline & P2 & 200,0 & 81,0 & 26,5 & 6,0 & 0 & 12,3 & 1,6 & 1,9 & 2,8 & 24,9 & 1,0 & 2,8 & 112,9 & 0,0 & 0,01 & 153,9 \\
\hline & P3 & 80,0 & 19,0 & 26,7 & 5,3 & 0 & 26,2 & 1,3 & 0,6 & 0,9 & nd & 1,0 & 0,5 & 106,8 & 0,0 & 0,07 & 75,8 \\
\hline & P4 & 260,0 & 116,0 & 26,6 & 4,7 & 0 & 28,6 & 6,3 & 3,9 & 7,4 & 30,0 & 3,0 & 6,0 & 57,9 & 0,0 & 0,01 & 200,1 \\
\hline & P5 & 119,0 & 28,0 & 26,8 & 5,5 & 0 & 33,9 & 2,0 & 1,0 & 3,5 & 28,4 & 4,0 & 2,6 & 131,2 & 0,0 & 0,02 & 112,8 \\
\hline & P6 & 96,0 & 47,0 & 26,5 & 6,1 & 1 & 47,4 & 1,6 & 1,0 & 8,4 & 30,1 & 7,0 & 2,4 & 134,2 & 0,0 & 0,03 & 90,9 \\
\hline & P7 & 107,0 & 76,0 & 26,6 & 5,8 & 0 & 51,5 & 1,7 & 0,5 & 9,0 & 22,7 & 2,0 & 2,1 & 97,5 & 0,0 & 0,05 & 101,4 \\
\hline & $\begin{array}{c}P \\
\text { moy }\end{array}$ & 168,8 & 72,1 & 26,6 & 5,6 & 0,1 & 32,1 & 2,3 & 1,7 & 5,6 & 28,3 & 4,6 & 3,2 & 108,9 & $\mathbf{0 , 0}$ & $\mathbf{0 , 0 3}$ & 129,9 \\
\hline & F1 & 21,0 & 38,0 & 19,2 & 6,0 & 0,0 & 24,0 & 7,1 & 0,3 & 0,1 & 0,1 & 5,2 & 1,3 & 6,8 & 0,1 & 0,02 & 21,8 \\
\hline & F2 & 87,8 & 44,1 & 21,0 & 5,8 & 0,2 & 24,0 & 7,8 & 0,8 & 0,1 & 0,2 & 6,0 & 3,1 & 4,7 & 0,1 & 0,00 & 83,2 \\
\hline & F3 & 118,0 & 50,0 & 23,0 & 6,2 & 0,0 & 17,0 & 6,1 & 0,5 & 0,2 & 1,8 & 7,0 & 4,6 & 7,4 & 0,1 & 0,05 & 111,8 \\
\hline & $\begin{array}{c}\text { F } \\
\text { moy }\end{array}$ & 75,6 & 44,0 & 21,1 & 6,0 & $\mathbf{0 , 1}$ & 21,7 & 7,0 & 0,5 & 0,1 & 0,69 & 6,1 & 3,0 & 6,3 & $\mathbf{0 , 1}$ & $\mathbf{0 , 0 2}$ & 71,6 \\
\hline \multicolumn{2}{|c|}{$\begin{array}{c}\text { Normes } \\
\text { O.M.S }\end{array}$} & $<300$ & $\begin{array}{l}1500 \\
\text { Max }\end{array}$ & 25 & $\begin{array}{l}7- \\
9,0\end{array}$ & 5 & $\begin{array}{l}40- \\
200\end{array}$ & $\begin{array}{l}5- \\
50\end{array}$ & $\begin{array}{l}20- \\
150\end{array}$ & $\begin{array}{c}10- \\
20\end{array}$ & $\begin{array}{l}20- \\
200\end{array}$ & $\begin{array}{l}10- \\
200\end{array}$ & $\begin{array}{c}25- \\
40\end{array}$ & 150 & 0,05 & $\begin{array}{l}0,05 \\
-0,3\end{array}$ & \\
\hline \multirow{5}{*}{ 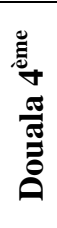 } & PI 2 & 62,0 & 30,1 & 26,2 & 6,8 & 1,0 & 17,9 & 0,6 & 1,2 & 1,8 & nd & $\mathbf{0 , 0}$ & 0,5 & 54,9 & 0,0 & $\mathbf{0 , 0 0}$ & 58,8 \\
\hline & P1 & 300,0 & 250,0 & 26,2 & 5,4 & 0,0 & 20,7 & 1,7 & 3,9 & 16,0 & 19,5 & 17,0 & 2,4 & 79,3 & 0,0 & 0,10 & 230,9 \\
\hline & $\mathbf{P 2}$ & 722,0 & 366,0 & 26,5 & 6,7 & 87,0 & 19,3 & 4,1 & 7,0 & 24,9 & 30,2 & 7,0 & 0,0 & 951,0 & 0,0 & 0,80 & 516,9 \\
\hline & P3 & 482,0 & 242,0 & 26,7 & 6,9 & 11,0 & 8,3 & 2,9 & 4,7 & 10,2 & 35,5 & 1,0 & 0,4 & 549,0 & 0,1 & 0,50 & 345,1 \\
\hline & P4 & 492,0 & 247,0 & 26,7 & 6,7 & 75,0 & 15,2 & 4,9 & 2,6 & 7,4 & nd & 47,0 & 0,0 & 518,5 & 0,0 & 0,90 & 352,3 \\
\hline
\end{tabular}


A. KOPA NJUEYA et al. / Int. J. Biol. Chem. Sci. 6(4): 1874-1894, 2012

\begin{tabular}{|c|c|c|c|c|c|c|c|c|c|c|c|c|c|c|c|c|}
\hline P5 & 380,0 & 276,0 & 26,8 & 5,8 & 23,0 & 38,7 & 2,6 & 3,7 & 13,8 & 17,1 & 22,0 & 1,3 & 170,8 & 0,1 & 0,25 & 272,1 \\
\hline P6 & 412,0 & 305,0 & 26,4 & 6,8 & 0,0 & 39,2 & 2,8 & 7,1 & 12,6 & 25,3 & 12,0 & 0,2 & 94,6 & 0,0 & 0,09 & 294,9 \\
\hline P7 & 507,0 & 312,0 & 26,6 & 6,9 & 1,0 & 26,9 & 2,6 & 6,2 & 9,8 & 29,1 & 4,0 & 1,5 & 112,9 & 0,0 & 0,14 & 362,9 \\
\hline $\begin{array}{c}\mathbf{P} \\
\text { moy }\end{array}$ & 470,7 & 285,4 & 26,6 & 6,5 & 28,1 & 24,1 & 3,1 & 5,0 & 13,5 & 22,4 & 15,7 & 0,8 & 353,8 & $\mathbf{0 , 0}$ & $\mathbf{0 , 4 0}$ & $\mathbf{3 3 7 , 0}$ \\
\hline F2 & 160,0 & 80,3 & 23,7 & 8,7 & 0,0 & 26,3 & 4,9 & 1,0 & 2,8 & 12,0 & 20,0 & 0,7 & 42,0 & 0,1 & 0,04 & 151,6 \\
\hline F3 & 80,6 & 72,0 & 23,5 & 7,6 & 0,0 & 18,9 & 3,0 & 0,7 & 0,9 & 10,1 & 12,0 & 0,7 & 12,5 & 0,7 & 0,02 & 76,4 \\
\hline F11 & 274,0 & 137,0 & 23,8 & 7,8 & 0,0 & 6,0 & 4,0 & 2,1 & 2,4 & 10,0 & 8,0 & 0,7 & 8,0 & 0,1 & 0,06 & 210,9 \\
\hline F7 & 423,0 & 212,0 & 23,3 & 6,8 & 0,0 & 8,0 & 2,6 & 1,1 & 0,6 & 9,5 & 21,6 & 2,1 & 11,0 & 0,2 & 0,03 & 302,8 \\
\hline $\begin{array}{c}\text { F } \\
\text { moy }\end{array}$ & 234,4 & 125,3 & 23,6 & 7,7 & $\mathbf{0 , 0}$ & 14,8 & 3,6 & 1,2 & 1,7 & 10,4 & 15,4 & 1,1 & 18,4 & 0,1 & 0,04 & 180,1 \\
\hline
\end{tabular}

Tableau 2: Evolution des différents rapports entre les alcalins et les alcalino-terreux dans les différentes catégories d'eau.

\begin{tabular}{|c|c|c|c|c|c|}
\hline Loc. & type & $\mathrm{Mg}^{2+} / \mathrm{Ca}^{2+}$ & $\mathrm{Na}^{+} / \mathbf{K}^{+}$ & $\left(\mathrm{Mg}^{2+}+\mathrm{Ca}^{2+}\right) /\left(\mathrm{Na}^{+}+\mathrm{K}^{+}\right)$ & I.E.B \\
\hline \multirow{3}{*}{$\begin{array}{l}m \\
\frac{\pi}{\pi} \\
\frac{0}{0} \\
0\end{array}$} & Pluie & 0,06 & 0,68 & 8,78 & nd \\
\hline & Puits & 0,07 & 0,31 & 4,70 & 0,74 \\
\hline & Forage & 0,32 & 3,79 & 42,81 & 0,03 \\
\hline \multirow{3}{*}{ 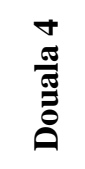 } & Pluie & 0,04 & 0,69 & 6,08 & nd \\
\hline & Puits & 0,13 & 0,37 & 1,46 & 0,17 \\
\hline & Forage & 0,25 & 0,74 & 6,35 & 0,72 \\
\hline
\end{tabular}


A. KOPA NJUEYA et al. / Int. J. Biol. Chem. Sci. 6(4): 1874-1894, 2012

Tableau 3: Evolution des caractéristiques microbiologiques des eaux de puits en fonction des saisons.

\begin{tabular}{|c|c|c|c|c|c|}
\hline \multirow{2}{*}{ Localité } & \multirow{2}{*}{ Echantillons } & \multirow{2}{*}{ Paramètres } & \multicolumn{3}{|c|}{ Période d'échantillonnage } \\
\hline & & & Février & Août & Novembre \\
\hline \multirow{6}{*}{ 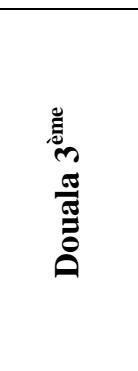 } & \multirow{3}{*}{ ZNJ03 } & C T. (UFC/100 ml) & 327 & 54 & 230 \\
\hline & & C F. (UFC/100 ml) & 290 & 16 & 100 \\
\hline & & S F. (UFC/100 ml) & 402 & 98 & 150 \\
\hline & \multirow{3}{*}{ ZNJ05 } & C T. (UFC/100 ml) & 597 & 37 & 200 \\
\hline & & C F. (UFC/100 ml) & 409 & 08 & 190 \\
\hline & & S F. (UFC/100 ml) & 321 & 43 & 130 \\
\hline \multirow{6}{*}{ 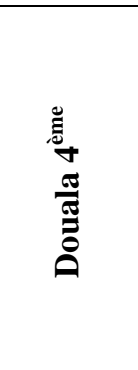 } & \multirow{3}{*}{ MNJ02 } & C T. (UFC/100 ml) & 1657 & 48 & 440 \\
\hline & & C F. (UFC/100 ml) & 1200 & 19 & 40 \\
\hline & & S F. (UFC/100 ml) & 3047 & 28 & 180 \\
\hline & \multirow{3}{*}{ MNJ04 } & C T. (UFC/100 ml) & 3645 & 17 & 170 \\
\hline & & C F. (UFC/100 ml) & 2200 & 0 & 23 \\
\hline & & S F. (UFC/100 ml) & 2689 & 23 & 140 \\
\hline
\end{tabular}




\section{DISCUSSION}

$\mathrm{Au}$ regard des résultats de l'analyse morphostructurale, l'aspect structural du bassin de Douala serait effectivement contrôlé par l'action de la tectonique panafricaine et de la Ligne du Cameroun (Njueya Kopa, 2011); ce qui signifie que le tracé du réseau hydrographique en place suivrait dans la majeure partie des cas les fractures ayant affecté le substratum en profondeur.

En prenant en compte la description des différentes argiles sur le terrain et en procédant par analogie aux argiles décrites et caractérisées par Ngueutchoua (1996) et Hieng (2003), on pourrait dire que ces formations argileuses sont riches respectivement en minéraux du groupe de la kaolinite, smectite/montmorillonite et du groupe de l'illite. Les argiles grises quant à elles, se rapprocheraient des horizons à gley identifiés par Ngueutchoua (1996) ou des argiles grises à gley réduit tel qu'identifié par Hieng (2003).

La détermination des caractéristiques hydrodynamiques des aquifères profonds permet d'estimer la porosité efficace moyenne entre 15 et $25 \%$; ceci par comparaison aux valeurs proposées par l'US. (Castany, 1982). Les différentes valeurs de perméabilité obtenues montrent que les différents aquifères pourraient avoir une forte productivité d'où ils seraient bien alimentés en eau.

L'étude hydrogéochimique montre une croissance des ions calcium, sodium, potassium, chlorures, bicarbonates et du fer. Ceci peut s'expliquer par le manque d'épaisses couches d'argiles ainsi que par l'altération des minéraux sombres (surtout ferromagnésiens) contenus dans les sables. Cependant, la diminution des différentes concentrations de ces ions pourrait être attribuée à la présence d'épaisses couches d'argiles qui pourraient jouer un rôle fixateur à travers l'adsorption ou l'absorption des éléments comme le potassium ou le calcium. La croissance des ions magnésiums pourrait s'expliquer par le fait que les argiles observées pourraient libérer les ions magnésium qu'elles contiennent pour fixer les ions comme le calcium ou le sodium. Cette croissance peut être due à l'altération progressive des minéraux ferromagnésiens observés dans les fractions sableuses de la lithologie.

La comparaison des valeurs moyennes de la conductivité montre que les eaux de Douala $3^{\text {ème }}$ dans l'ensemble, ont une minéralisation faible à moyenne et sont appropriées pour l'AEP ; tandis que les eaux de Douala $4^{\text {ème }}$ dans l'ensemble auraient une minéralisation moyenne à importante et ne seraient pas appropriées pour l'AEP conformément aux normes OMS. En ce qui concerne les STD et la turbidité, les valeurs pour la localité de Douala $3^{\text {ème }}$ sont en dessous des valeurs directrices fixées par l'OMS (1996) tandis qu'à Douala $4^{\text {ème }}$, les STD sont conformes aux prescriptions de l'OMS pourtant, tel n'est pas le cas pour la turbidité qui est largement au dessus du maximum fixé par l'OMS. Ainsi, ces eaux méritent d'être filtrées avant tout usage. Les températures des eaux sont proches de celle régnant en surface, cela confirmerait le caractère libre de ces nappes qui subiraient l'influence directe des eaux d'infiltration le principal moyen de recharge de ces nappes. Le fer semble conforme dans l'ensemble dans les eaux superficielles de Douala $3^{\text {ème }}$ et non conforme dans celles de Douala $4{ }^{\text {ème }}$. Donc les eaux des puits de Douala $3{ }^{\text {ème }}$ sont de qualité acceptable vis-à-vis du fer ce qui n'est pas le cas pour celles des puits de Douala $4^{\text {ème }}$ dans l'ensemble. Quant aux eaux des nappes profondes, elles sont de bonne qualité en ce qui concerne les alcalinoterreux.

$\mathrm{Au}$ terme de cette analyse des paramètres chimiques, les eaux des nappes profondes semblent potables en tout point de vue et peuvent être utilisées dans l'AEP.

L'augmentation progressive du rapport entre alcalino-terreux de la surface en profondeur, permet d'émettre l'hypothèse selon laquelle d'une part, les argiles fixeraient les ions calcium et libèreraient les ions magnésium et d'autres part, que l'excès de magnésium proviendrait des processus d'altération des minéraux ferromagnésiens observés dans les fractions sableuses identifiées lors de la réalisation des coupes lithologiques.

La tendance du rapport entre alcalinoterreux et alcalin à décroître puis à croître pourrait traduire dans l'ensemble, la tendance 
des eaux des deux zones à absorber les alcalins et à libérer les alcalino terreux. Cependant cette absorption serait faible dans les eaux des nappes superficielles et élevée dans les eaux des nappes profondes. Le diagramme de Hendry et Wassenaar (2000) permet de traduire cet enrichissement en alcalino terreux de l'ensemble des eaux des deux localités (Figure 4).

La baisse du rapport entre les alcalins dans l'ensemble des deux zones lorsqu'on passe des eaux de pluie aux eaux de puits, pourrait s'expliquer par le fait qu'on ait une fixation du sodium en surface et une libération $\mathrm{du}$ potassium. Cependant, son augmentation observée lorsqu'on passe des eaux de puits aux eaux de forage, pourrait traduire une rétention des ions potassium par les argiles et une libération des ions sodium au fur et à mesure qu'on évolue en profondeur. Ceci peut se justifier par le fait que le potassium a un rayon atomique $\left(\mathrm{R}_{\mathrm{K}+}=2,35 \AA\right)$ supérieur à celui du sodium $\left(\mathrm{R}_{\mathrm{Na}+}=1,91 \AA\right)$ d'où il sera préférentiellement capté par les argiles (surtout ceux de type 2/1 ou 2/1/1 comme les smectites, les montmorillonites ou les illites) pour assurer la stabilité de leur structure.

L'I.E.B qui donne des valeurs supérieures à zéro dans les différentes catégories d'eaux souterraines justifie le fait que les alcalins $\left(\mathrm{Na}^{+}\right.$et $\left.\mathrm{K}^{+}\right)$de l'eau sont échangés (substitués) par les alcalino-terreux $\left(\mathrm{Mg}^{2+}\right.$ et $\left.\mathrm{Ca}^{2+}\right)$ des terrains traversés. Cependant, on constate que cette substitution est plus prononcée dans les eaux de puits de Douala $3^{\text {ème }}$ que dans les eaux de puits de Douala $4^{\text {ème; }}$ tandis que l'on observe un comportement opposé en comparant l'IEB des eaux de forage des deux localités. Ces différents échanges comme le fait remarquer Bouziane et Labadi (2009), s'expliquent par le processus d'inter action eau - roche.

De par leur conductivité, les eaux des nappes superficielles dans les deux zones d'études et de façon plus générale dans le bassin de Douala, sont plus minéralisées que les eaux des nappes profondes. Ce phénomène peut s'expliquer par l'action des argiles et sables situés en dessous des aquifères superficiels qui fixeraient les ions et certains complexes (diminuant ainsi la conductivité et la turbidité) en jouant un rôle de filtre naturel.
$\mathrm{Au}$ regard des concentrations des différents paramètres, on se rend compte que la minéralisation des eaux des nappes superficielles est liée aux ions bicarbonates; cependant la minéralisation des eaux des nappes profondes est liée majoritairement aux ions calcium.

Le diagramme de Gibbs (1970) montre trois domaines auxquels appartiennent les différentes catégories des eaux analysées (Figure 5). Le premier domaine est celui des eaux des forages des deux localités qui montrent une minéralisation influencée par les processus d'interaction eau - roche (I.E.R).

Le deuxième domaine montre que l'ensemble des eaux des puits de Douala $3^{\text {ème }}$ et seulement une partie des eaux de Douala $4^{\text {ème }}$ auraient une minéralisation influencée à la fois par les processus d'interaction eauroche et par les phénomènes d'évaporation.

Le troisième domaine est celui d'une partie des eaux de puits de Douala $4{ }^{\text {ème }}$ qui montrent une minéralisation influencée par l'évaporation et surtout par l'action de la mer, pour les échantillons $\left(\mathrm{P}_{2}, \mathrm{P}_{3}\right.$ et $\left.\mathrm{P}_{4}\right)$ se trouvant à proximité du Wouri (Cf. Figure 1). À partir de ce résultat, on peut déduire que toutes les eaux situées à proximité du Wouri subissent une influence de la mer dans leurs compositions physico-chimiques.

La détermination des minéraux normatifs en équilibre avec les différentes catégories d'eaux souterraines à partir des diagrammes d'activité alcalin - alcalino terreux à trois dimensions (Figure 6), montre que les eaux sont en équilibre avec cinq principaux minéraux (par ordre d'abondance) que sont: la pyrophyllite, la montmorillonite, la chlorite, la dolomite et la laumontite.

La présence des minéraux argileux tels que la pyrophyllite dans toutes les eaux de puits et à moindre mesure de la montmorillonite dans les eaux de certains forages, permet de dire que les processus d'altération dominants infuençant sur la minéralisation des eaux est l'hydrolyse à travers la bisiallitisation responsable de la mise en place de la pyrophyllite $\left[\mathrm{Al}_{2} \mathrm{Si}_{4} \mathrm{O}_{10}(\mathrm{OH})_{2}\right]$ et la de la montmorillonite. Cette évolution des processus d'altération de la surface à la profondeur où domine la bisiallitisation peut se justifier par le fait qu'en 
profondeur le milieu est plus confiné qu'en surface si l'on considère le contexte géologique du milieu.

\section{Hypothèses sur l'origine et les processus de mise en solution de certains ions}

Les différents ions observés dans les eaux de puits et de forage dans les arrondissements de Douala $3^{\text {ème }}$ et de Douala $4^{\text {ème }}$, seraient issus de l'altération des roches du métamorphiques ou magmatiques situées en bordure du bassin et riches en minéraux ferromagnésiens. L'évolution de la minéralisation lorsqu'on passe des eaux de pluie aux eaux de puits, montre qu'au cours de son infiltration, l'eau se charge de certains éléments contenus dans les formations qu'elle traverse. Cependant, la diminution de la minéralisation lors du passage des eaux de puits aux eaux de forage montre que les argiles contenues dans le sol retiennent certains éléments chimiques.

\section{Cas des alcalins}

Les alcalins observés dans les eaux des puits et des forages pourraient provenir de l'altération des minéraux renfermant le potassium et le sodium contenus dans les roches ayant donné naissance aux sédiments de remplissage du bassin. Il s'agit par exemple des minéraux comme l'orthose et l'albite qui par bisiallitisation peuvent donner la pyrophyllite. Ceci peut se faire conformément aux équations ci-dessous :

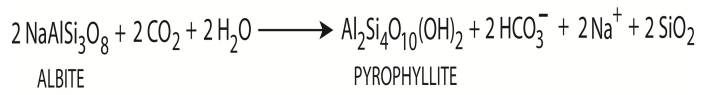

$$
\underset{\text { ORTHOSE }}{2 \mathrm{KAISi}_{3} \mathrm{O}_{8}+2 \mathrm{CO}_{2}+2 \mathrm{H}_{2} \mathrm{O} \longrightarrow} \underset{\substack{\mathrm{Al}_{2} \mathrm{~S}_{4} \mathrm{O}_{10}\left(\mathrm{OH}_{2} \\\right. \text { PYROPHYLLTE }}}{\longrightarrow}+2 \mathrm{HCO}_{3}^{-}+2 \mathrm{~K}^{+}+2 \mathrm{SiO}_{2}
$$

La présence d'un excès de potassium par rapport au sodium peut s'expliquer par le fait que les roches responsables de la mise en place des sédiments, devraient être très riches en potassium. Ceci se justifie par le fait que les roches de la CPAC sont calcoalcalines à tendance hyperpotassique (Njonfang et al., 2006).

\section{Cas des alcalinoterreux}

La présence du calcium peut s'expliquer d'une part par l'altération des roches ayant des minéraux renfermant le calcium tels que les plagioclases. D'autre part vu le contexte géologique du milieu, on peut également penser qu'il peut provenir de l'altération de la calcite (ou des carbonates de calcium). Ceci peut par exemple s'effectuer conformément aux équations ci-dessous:

$\mathrm{CaCO}_{3}$ (calcite) $+\mathrm{H}_{2} \mathrm{O}+\mathrm{CO}_{2} \longrightarrow \mathrm{Ca}^{2+}+2 \mathrm{HCO}_{3}^{-}$

$$
\begin{aligned}
& \mathrm{CaAl}_{2} \mathrm{Si}_{2} \mathrm{O}_{8}+2\left(\mathrm{SiO}_{2}, \mathrm{CO}_{2}\right)+2 \mathrm{H}_{2} \mathrm{O} \longrightarrow \mathrm{Al}_{2} \mathrm{Si}_{4} \mathrm{O}_{10}\left(\mathrm{OH}_{2}+2 \mathrm{HCO}_{3}^{-}+\mathrm{Ca}^{2+}\right. \\
& \text { ANORTHTE PYROPHYLLTE }
\end{aligned}
$$

L'équation (2) est celle s'effectuant en condition de bisiallitisation et pouvant expliquer le fait qu'on ait la pyrophyllite en équilibre avec les eaux analysées.

Le magnésium quant à lui pourrait avoir trois origines: la première origine est qu'il proviendrait de l'altération des minéraux ferromagnésiens (phlogopite, amphibole, etc.); la seconde est qu'il pourrait provenir des processus de dissolution de la dolomite et le troisième processus serait le phénomène de substitution entre le magnésium libéré du talc et l'aluminium libéré des minéraux contenant l'aluminium dans les roches altérées. Tous ces phénomènes peuvent s'expliquer à travers les équations ci-dessous:

$$
\begin{aligned}
2 \mathrm{KMg}_{3} \mathrm{AlSi}_{3} \mathrm{O}_{10}(\mathrm{OH})_{2}+\mathrm{CO}_{2}+13 \mathrm{H}^{+}+2 \mathrm{H}_{2} \mathrm{O} \longrightarrow & \mathrm{Al}_{2} \mathrm{Si}_{2} \mathrm{O}_{5}\left(\mathrm{OH}_{4}+4 \mathrm{H}_{4} \mathrm{SiO}_{4}+2 \mathrm{~K}^{+}\right. \\
& +6 \mathrm{Mg}^{2+}+\mathrm{HCO}_{3}^{-} \\
\mathrm{CaMg}\left(\mathrm{CO}_{3}\right)_{2}(\text { dolomite })+\mathrm{Ca}^{2+} \longrightarrow \mathrm{CaCO}_{3} \text { (calcite) }+\mathrm{Mg}^{2+} & (4) \\
\mathrm{Mg}_{3} \mathrm{Si}_{4} \mathrm{O}_{10}(\mathrm{OH})_{2}(\mathrm{talc})+\mathrm{Al}_{2} \mathrm{O}_{3}+6 \mathrm{H}^{+} \longrightarrow \mathrm{Al}_{2} \mathrm{Si}_{4} \mathrm{O}_{10}\left(\mathrm{OH}_{2}+3 \mathrm{Mg}^{2+}+3 \mathrm{H}_{2} \mathrm{O}\right. & \text { (5) }
\end{aligned}
$$

L'augmentation des proportions du magnésium au fur et à mesure que l'on progresse en profondeur, peut s'expliquer le plus probablement à travers l'équation (4) ou (5): car selon Ngueutchoua (1996), l'on a une augmentation du taux de calcite au fur et à mesure que l'on progresse en profondeur dans les profils pédologiques. Cette augmentation peut s'expliquer par ce phénomène de dissolution de la dolomite et formation des carbonates de calcium. 


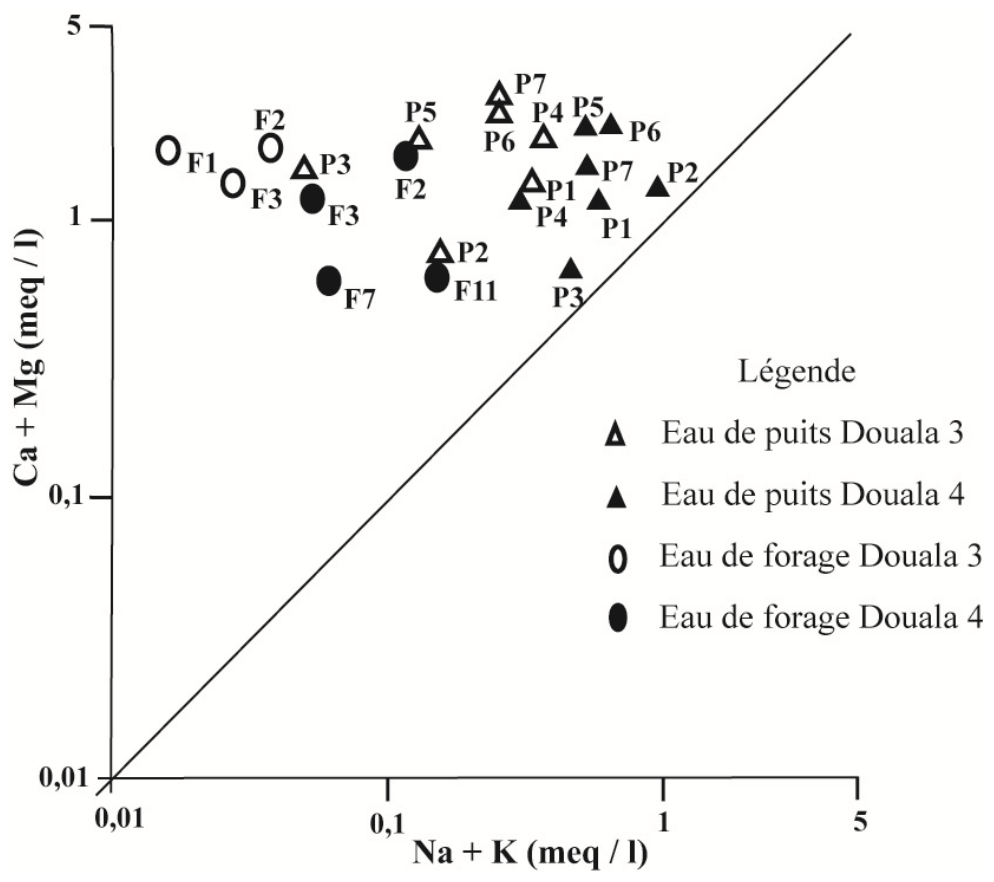

Figure 4: Diagramme de Hendry et Wassenaar donnant le comportement des différents types d'eau vis-à-vis des alcalins et des alcalino - terreux.

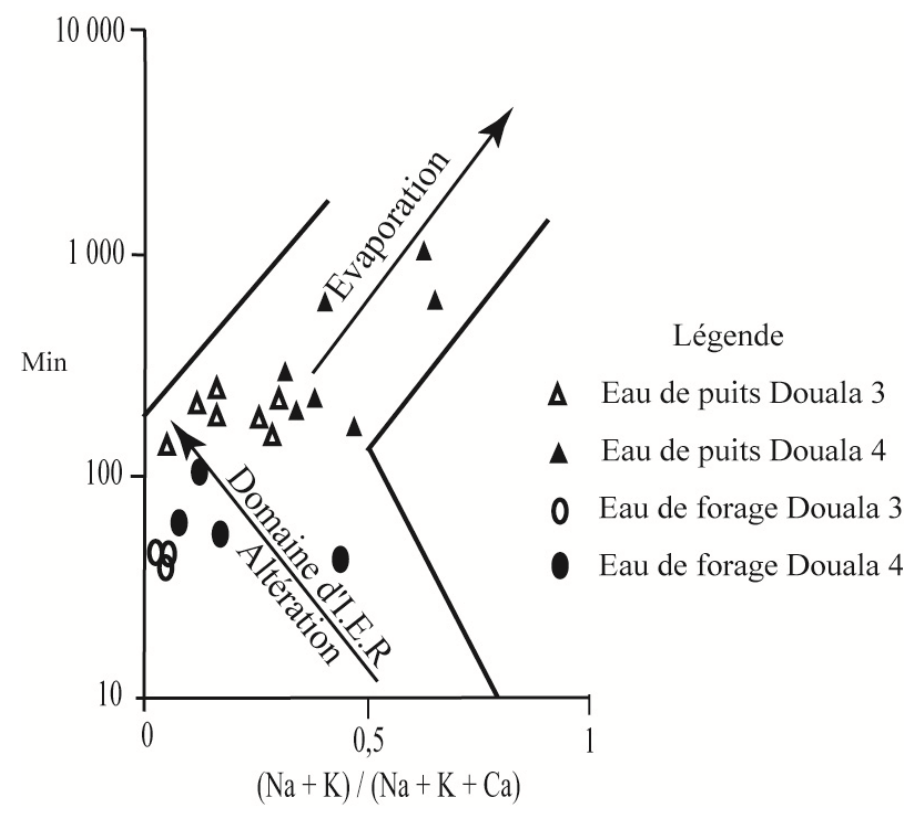

Figure 5: Diagramme de Gibbs traduisant l'origine de la minéralisation des différents échantillons d'eau souterraine. 


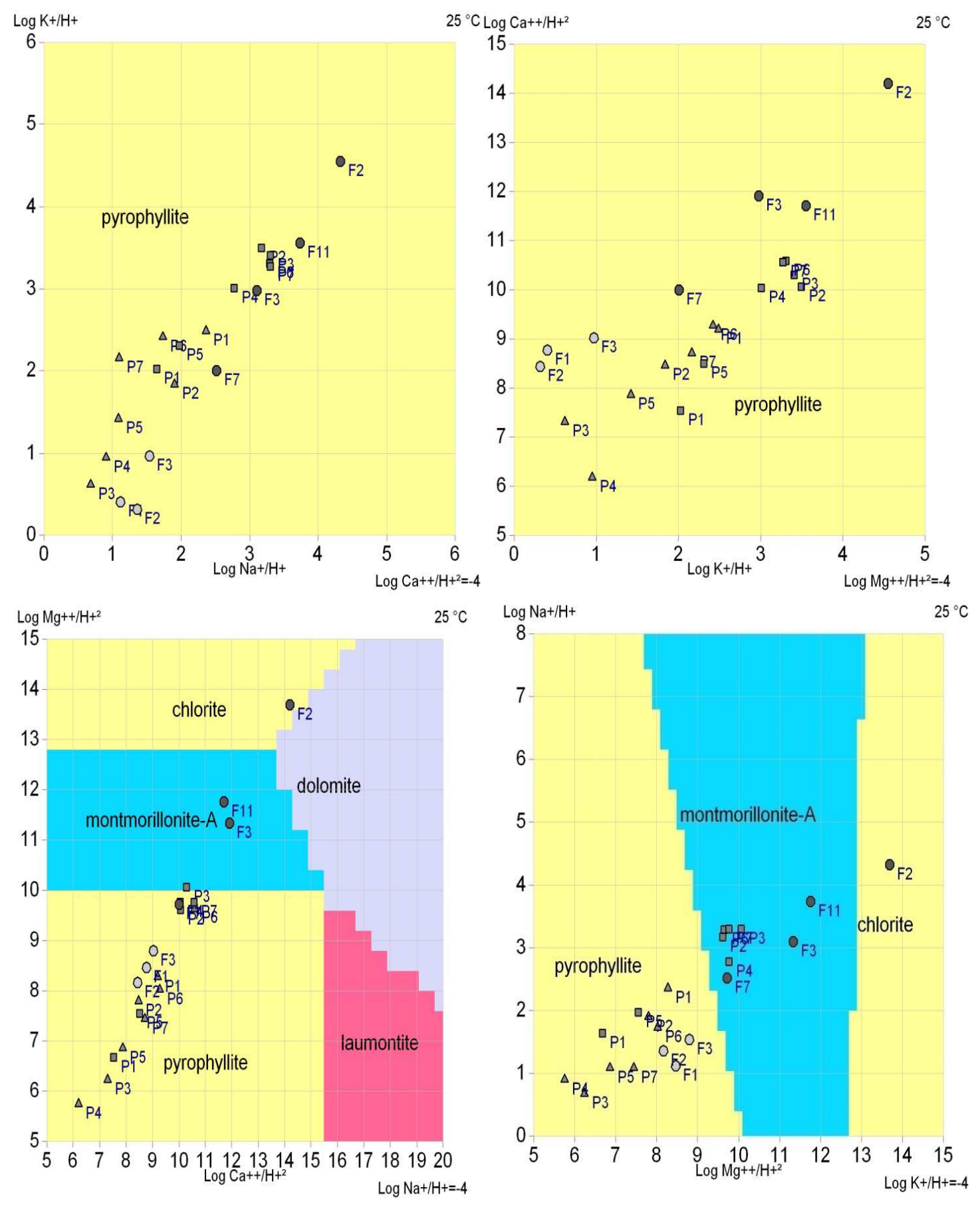

Figure 6: Diagramme d'activité alcalin-alcalino terreux donnant les principaux minéraux normatifs en équilibre avec les eaux souterraines analysées. 


\section{Cas des ions bicarbonates $\left(\mathrm{HCO}_{3}^{-}\right)$}

Les ions bicarbonates peuvent provenir de l'altération de certains minéraux (les feldspaths, la calcite...) ou de la combinaison entre le dioxyde de carbone atmosphérique ou contenu dans le sol avec les molécules d'eau au moment des précipitations. Cependant, il peut aussi provenir de l'action des microorganismes contenus dans le sol à travers la dégradation de la matière végétal. Ce dernier processus est le plus probable expliquant un taux élevé de bicarbonate dans les eaux des puits $\mathrm{P}_{2}, \mathrm{P}_{3}$ et $\mathrm{P}_{4}$ échantillonnées dans Douala $4^{\text {ème }}$ dans une ancienne zone de mangrove occupée aujourd'hui par les populations.

Cas du fer

Le fer provient de l'hydrolyse des minéraux riches en fer comme les biotites, les pyroxènes ou les olivines contenus soit dans les formations du socle ou les formations de la L.C qui se sont altérées pour produire les différents sédiments. Le fer une fois libéré de ces minéraux se trouve sous sa forme dissoute $\left(\mathrm{Fe}^{2+}\right)$ et une fois au contact de l'air s'oxyde (en $\mathrm{Fe}^{3+}$ ) pour être sous forme hydraté insoluble (limonite) ayant une couleur rouge rouille. Ceci s'explique conformément à l'équation suivante :

$4 \mathrm{Fe}^{3+}+3 \mathrm{O}_{2}+\mathrm{H}_{2} \mathrm{O} \longrightarrow 2 \mathrm{Fe}_{2} \mathrm{O}_{3}, \mathrm{H}_{2} \mathrm{O}+12 \mathrm{e}^{-}$

\section{Evolution de la qualité bactériologique des eaux}

Il en ressort de l'analyse bactériologique des eaux obtenues en comparaison aux valeurs établies par l'OMS (1982) en vue d'un traitement probable et efficace, que les eaux des puits évoluent d'une qualité assez bonne en pleine saison pluvieuse, puis à une qualité moyenne à mi saison pluvieuse. Cependant, en saison sèche, on a une évolution vers les eaux de qualité suspecte à Douala $3^{\text {ème }}$ tandis qu'à Douala $4^{\text {ème }}$, la tendance est celle des eaux de qualité médiocre. En prenant en compte les prescriptions de l'OMS (1982), les eaux des puits dans le bassin de Douala nécessitent d'être surveillées et traitées impérativement avant toute consommation par les populations. De même il ressort de ces résultats, que pendant les saisons pluvieuses, on a un effet de dilution qui diminue la concentration des bactéries dans l'eau; ce qui peut fausser le jugement des populations ou des ingénieurs sanitaires sur les procédures à mettre en œuvre pour la potabilisation des eaux de puits.

\section{Conclusions}

Il ressort de cette étude menée sur l'évolution de la qualité des ressources en eaux souterraines dans le bassin de Douala sur les formations Quaternaires (Douala $4^{\text {ème }}$ ) et les formations Mio-pliocènes (Douala $3^{\text {ème }}$ ) de nombreux résultats sur le plan géologique, géophysique, hydrogéologique, hydrogéochimique et microbiologique.

Les études géologiques montrent que la tectonique panafricaine et la mise en place de la L.C ont influencé la mise en place des sédiments observés ainsi que le tracé du réseau hydrographique qui est parfois rectiligne et montre des directions variantes de $\mathrm{N} 70^{\circ} \mathrm{E}$ à $\mathrm{N} 80^{\circ} \mathrm{E}$ et de $\mathrm{N} 30^{\circ} \mathrm{E}$ à $\mathrm{N} 40^{\circ} \mathrm{E}$. La description des profils révèle la présence des horizons riches en fer et situés directement au dessus des nappes superficielles. De même, elle montre que la lithologie entre 0 et $100 \mathrm{~m}$ de profondeur est constituée des sables, argiles et grès qui de par leurs constitutions, joueraient un rôle de filtre sélectif influençant l'évolution de la qualité des eaux souterraines.

Les études géophysiques couplées aux résultats de foration, font ressortir la présence de deux principaux aquifères entre 0 et $100 \mathrm{~m}$ de profondeur. On distingue une nappe superficielle exploitée à travers les puits (de 0 à $13 \mathrm{~m}$ à Douala $4^{\text {ème }}$ et de 0 à $17 \mathrm{~m}$ à Douala $3^{\text {ème }}$ ) et une nappe profonde exploitée au moyen des forages (de 38 à $73 \mathrm{~m}$ à Douala $4^{\text {ème }}$ et de 20 à $40 \mathrm{~m}$ à Douala $3^{\text {ème }}$ ). Dans la localité de Douala $4{ }^{\text {ème }}$, les aquifères profonds semblent être continus et hétérogènes ce qui semble ne pas être le cas dans la zone de Douala $3^{\text {ème }}$. Les aquifères mio-pliocènes sont constitués des sables fins à moyens souvent argileux (niveau superficiel) et des sables fins à moyens ainsi que des grès ferrugineux (niveau profond); tandis que les aquifères quaternaires sont constitués majoritairement de sable moyens à grossiers souvent graveleux.

L'étude hydrogéologique, révèle que la région a un fort potentiel. Les formations aquifères présentent une bonne perméabilité 
qui peut s'apprécier à l'aide des paramètres hydrodynamiques tel que le coefficient de perméabilité qui présente une moyenne de 2,7 x $10^{-2} \mathrm{~m} / \mathrm{s}$ et une porosité moyenne variante de 15 à $25 \%$. La transmissivité varie dans l'ensemble entre $6,354 \times 10^{-2} \mathrm{~m}^{2} / \mathrm{s}$ et 0,165 $\mathrm{m}^{2} / \mathrm{s}$ pour les deux localités.

Sur le plan hydrogéochimique, les eaux des nappes souterraines dans le bassin de Douala montrent une évolution quasi similaire en dépit de leurs âges différents. On a une évolution du faciès bicarbonaté calcique (pour les eaux des pluies), au faciès bicarbonaté calcique et magnésien (pour les eaux des puits) et enfin au faciès chloruré et sulfaté calcique et magnésien pour les eaux de forages. Les eaux des puits présentent une forte minéralisation liée aux ions bicarbonates; tandis que les eaux des nappes profondes ont une faible minéralisation dominée par les ions calcium. La minéralisation des eaux dans l'ensemble est influencée par les processus d'interaction eau - roche. Néanmoins, signalons l'influence de la mer à travers les eaux du Wouri dans les eaux des nappes superficielles situées à proximité de la mer.

Dans l'ensemble, les eaux des nappes profondes (forages) sont de bonne qualité tant sur le plan physico-chimique ainsi que bactériologique et respectent les normes OMS: ce qui montre que les couches d'argiles situées au-dessus de ces aquifères jouent un rôle protecteur et absorbent les polluants s'infiltrant verticalement dans le sol. Cependant, les eaux des nappes superficielles (eau de puits) présentent une bonne qualité sur le plan physico-chimique excepté leur taux élevé en bicarbonate et en fer à Douala $4^{\text {ème }}$. Sur le plan bactériologique, les eaux des nappes superficielles montrent une qualité qui varie en fonction des saisons. En saison sèche, elles sont de qualité suspecte à Douala $3^{\text {ème }}$ et de qualité médiocre à Douala $4{ }^{\text {ème }}$. Cependant, en saison pluvieuse, elles sont de qualité assez bonne dans les eaux superficielles des deux zones.

\section{REMERCIEMENTS}

Nous adressons nos remerciements à la société GEOBASE SARL qui nous a aidé dans l'acquisition des données géophysiques et leur mise en valeur, ainsi que des données pétrographiques en nous acceptant sur ses chantiers pour la réalisation de ce travail.

\section{REFERENCES}

Bouziane MT, Labadi A. 2009. Les eaux profondes de Biskra (Algérie). European Journal of Scientific Research, 25(4): 526-537.

BUCREP. 2010. Rapport final $3^{\text {ème }}$ recensement général de la population et de l'habitat au Cameroun. Répartition de la population résidant dans la province du Littoral par Département et par Arrondissement/District. Yaoundé, Cameroun, 45-47.

Castany G. 1982. Principes et Méthodes de l'Hydrogéologie ( $2^{\mathrm{ème}}$ édn). Collection Dunod Université - Bordas : Paris.

Djeuda TBH. 1987. Géologie et hydrogéologie d'un secteur de la zone mobile d'Afrique centrale région de Poli, Nord Cameroun. Thèse Doct., Univ. Grenoble I, Grenoble I, p.304+annexes.

Djuikom E, Temgoua E, Jugnia LB, Nola M, Baane M. 2009. Pollution bactériologique des puits d'eau utilisés par les populations dans la Communauté Urbaine de Douala - Cameroun. International Journal of Biological and Chemical Sciences, 3(5): 967-978.

Dumort JC. 1968. Carte géologique de reconnaissance de la république fédérale du Cameroun au 1/500000. Direction des mines et de la géologie, feuille NB 32 SE 028.

El Achheb A, Mania J, Mudry J. 2001. Processus de salinisation des eaux souterraines dans le bassin Sahel Doukkala (Maroc occidental). First International Conference on Saltwater Intrusion and Coastal Aquifers Monitoring, Modeling, and Management, Essaouira, Moroco, 23 25 april, 2001.

Fehdi C, Boudoukha A, Rouabhia A, Salameh E. 2009. Caractérisation hydrogéochimique des eaux souterraines du complexe aquifère Morsott-Laouinet (Région Nord de Tébessa, Sud-Est algérien). Afrique SCIENCE, $\mathbf{0 5}(2)$ : $217-$ 231. 
Gibbs RJ. 1970. Mechanisms controlling worlds water chemistry. Science, 170: 1088-1090.

Hendry MJ, Wassenaar LI. 2000. Controls on the distribution of majors ions in pore waters of a thick surficial aquitard. Water Resources Research, 36: 503-513.

Hieng IO. 2003. Etude des Paramètres Géotechniques des Sols du Cameroun. Editions CLE : Yaoundé, Cameroun.

INS. 2005. Annuaire Statistique du Cameroun 2004. Direction Nationale de la Statistique et de la Comptabilité Nationale : Yaoundé, Cameroun.

Koul'a NRT. 2006. Contribution du forage de Koumassi dans l'amélioration de l'approvisionnement en eau par la Société Nationale des Eaux du Cameroun à Douala. Mémoire d'ingénieur agronome Univ. Dschang, Dschang-Cameroun, p. 74.

Kreisel W. 1991. Water quality and health. Water Science Technology, 23: 201-209.

Leclerc H. 1986. Pathologie liée à l'usage de l'eau. Aqua., 6: 329 - 333.

Manga M. 2001. Using spring to study groundwater flow and active geologic process. Review Earth Planet Sciences, 29: 201-208.

Martin G. 1979. Alimentation de la ville de Douala à partir des eaux souterraines. Etudes et Travaux BRGM, p. 143.

Mouafo G. 2010. Influence des structures géologiques sur la qualité des eaux dans les hautes terres de l'ouest - Cameroun : cas de la région de Bamendjing et ses environs. Thèse Master of Sciences, Univ. Dschang, Dschang-Cameroun, p. 90.

Ngueutchoua G. 1996. Etude des faciès et environnements sédimentaires du quaternaire supérieur du plateau continental camerounais. Thèse de
Doctorat Univ. Perpignan4, Perpignan, p. 288.

Njike Ngaha PR. 1984. Contribution a l'étude géologique, stratigraphique et structurale de la bordure du bassin Atlantique du Cameroun. Thèse Doct. $3^{\text {ème }}$ cycle, Univ. Yaoundé I, Yaoundé-Cameroun, p. 131.

Njueya Kopa A. 2011. Etude de l'évolution de la qualité des eaux souterraines dans le bassin de Douala: cas des aquifères sur formations quaternaires à Douala $4{ }^{\text {ème }}$ et tertiaires à Douala $3^{\text {ème }}$. Thèse Master of Sciences, Univ. Dschang, DschangCameroun, p. 106.

Njonfang E, Ngako V, Kwékam M, Affaton P. 2006. Les orthogneiss calco- alcalins de Fouban: témoins d'une zone interne de marge active Panafricaine en cisaillement. C.R. Géoscience, 338: 606616.

Nono A, Likeng JDH, Wabo H, Tabue YG, Biaya S. 2009. Influence de la nature lithologique et des structures géologiques sur la qualité et la dynamique des eaux souterraines dans les hauts plateaux de l'Ouest-Cameroun. International Journal of Biological and Chemical Sciences, 3(2): 218-239.

OMS. 1982. Normas Internationales Para el agua Potable. OMS: Ginebra; 218.

OMS. 1996. Lignes Directives sur la Qualité de l'Eau de Boisson. (2 édn, Vol 2). OMS; 26.

Regnoult J. 1986. Synthèse géologique du Cameroun. Ministère des Mines, Yaoundé-Cameroun, 119.

Temgoua E, Djeuda THB, Tanawa E, Guenat C, Pfeifer H-R. 2005. Groundwater fluctuations and footslope ferricrete soils in the humid tropical zone of Southern Cameroon. Hydrological Process, 19: 3097-3111. 